\title{
Associative structure of integrated temporal relationships
}

\author{
Cody W. Polack • Mikael Molet • Gonzalo Miguez • \\ Ralph R. Miller
}

Published online: 16 August 2013

(C) Psychonomic Society, Inc. 2013

\begin{abstract}
According to the temporal-coding hypothesis (TCH; Savastano \& Miller, Behavioural Processes 44:147-162, 1998), acquired associations include temporal information concerning the interval between the associated elements. Moreover, the TCH posits that subjects can integrate two independently acquired associations that share a common element (e.g., S2-S1 and S1-US), which results in the creation of a third association with its own temporal relationship (S2-US). Some evidence has suggested that such temporal integration occurs at the time of testing (Molet, Miguez, Cham, \& Miller, Journal of Experimental Psychology: Animal Behavior Processes 38:369-380, 2012). Here we report two fear-conditioning experiments with rats conducted to identify the associative structure of the integrated temporal relationship. The goal was to distinguish between two possible associative structures that could exist following an initial test on which temporal integration occurs: (1) Conditioned responding to S2 on subsequent tests could be the result of recurring successive activation of two independently learned temporal maps that remain independently stored in memory (i.e., S2-S1 plus S1-US). (2) Temporal integration at the moment of initial testing could result in the formation of a direct S2-US (or S2-response) temporal map. Integration was found to occur at test and to produce a new association that was independent of associations with the common element (S1). However, the associative status of S1 appeared to modulate whether or not the new association with S2 was US-specific (S2-US) or directly activated a fear response (S2-response).
\end{abstract}

C. W. Polack $\cdot$ G. Miguez $\cdot$ R. R. Miller

State University of New York, Binghamton, NY, USA

M. Molet

University of Lille, Lille, France

R. R. Miller ( $\square)$

Department of Psychology, SUNY-Binghamton,

Binghamton, NY 13902-6000, USA

e-mail: rmiller@binghamton.edu
Keywords Sensory preconditioning - Temporal coding . Temporal integration $\cdot$ Associative structure

The temporal-coding hypothesis (TCH; see, e.g., Savastano \& Miller, 1998) is a set of hypotheses concerning how temporal information is used within any associative model. The tenets of the TCH can be summarized as follows: (a) Close contiguity between events is sufficient for the formation of an association; (b) the temporal relationship between the associated events (order and interval) is encoded as part of the association (i.e., temporal contiguity is not a mere catalyst for forming an association; see also Honig, 1981); (c) this temporal information plays a critical role in determining the topology, magnitude, and timing of the response elicited when one of the associates is subsequently presented; and (d) subjects can superimpose temporal relationships when the relationships share a common element, even when the relationships were independently acquired, thereby allowing for the expression of temporal relationships between cues that were never actually paired together (i.e., temporal integration).

Miller and colleagues (e.g., Matzel, Held, \& Miller, 1988) validated this view by showing that after subjects independently acquire two associations with a common element (e.g., S2S1 and S1-US in a sensory preconditioning paradigm), each with its own temporal relationship, they behave as if the two unique cues have a known temporal relationship, despite the cues' never having been paired (for reviews, see Arcediano \& Miller, 2002; Savastano \& Miller, 1998). Seemingly, the two associations are integrated to create a third association with its own temporal attributes (S2-US). This tenet is what makes the $\mathrm{TCH}$ unique in the timing literature, and is particularly evident in the case of temporal integration involving a backward association (US-S1; e.g., Arcediano, Escobar, \& Miller, 2003). In Arcediano et al.'s (2003) preparation, rats were administered S2-S1 pairings in a forward relationship with either a 5-s gap or no gap between the termination of S2 and the onset of S1 in Phase 1 (see Fig. 1, Groups Int and Con1, respectively). These treatments were followed by $\mathrm{S} 1-$ footshock US pairings presented in a backward relationship 


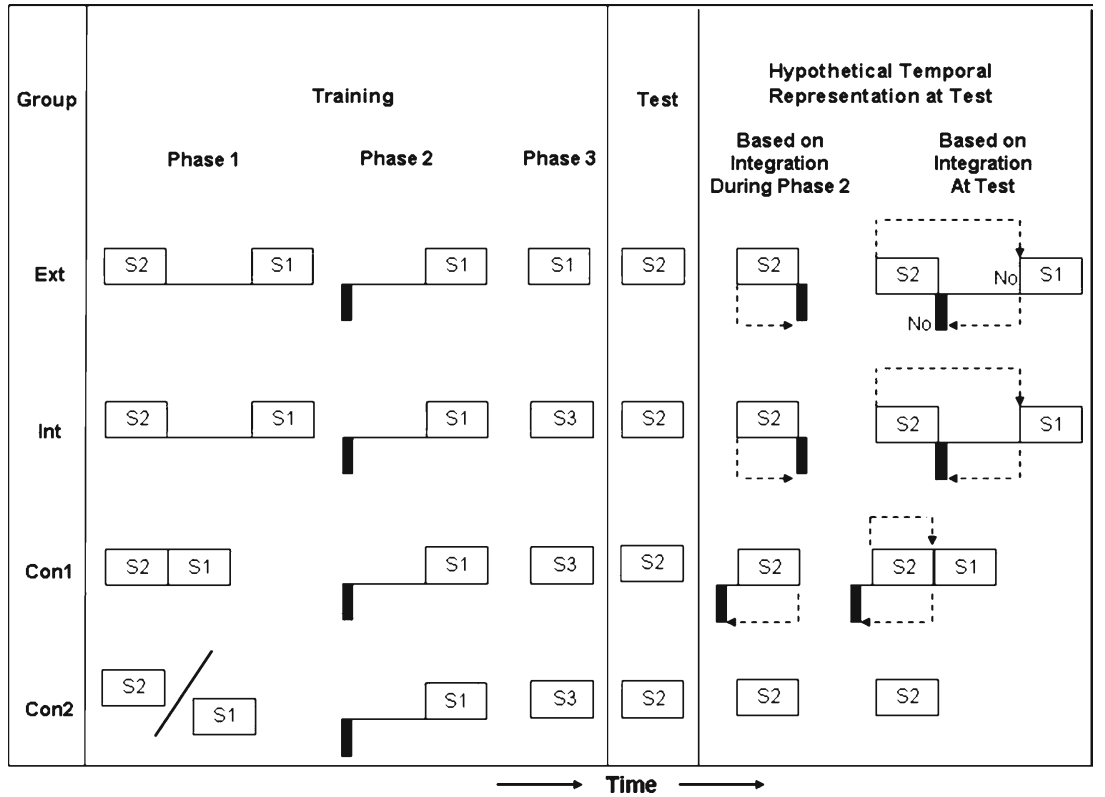

Fig. 1 Design of Molet et al. (2012, Exp. 1). The critical comparison for this design was between Group Int(egration) and Group Ext(inction). The authors observed less conditioned responding when $\mathrm{S} 1$ had been extinguished during Phase 3 (Group Ext) than when S1 had not been extinguished (Group Int). This indicates that integration occurred at the time of test because, otherwise, extinction of S1 would have been innocuous (i.e., US-S1), with a 4-s gap between termination of the US and the onset of S1 in Phase 2. When tested on S2, rats trained with a 5-s gap in Phase 1 showed a large amount of conditioned suppression, whereas rats trained with no gap in Phase 1 showed less conditioned suppression. Identical procedures, with highly similar results, were used in a subsequent study by Molet et al. (2012). According to the TCH, the rats had encoded the temporal relationships between $\mathrm{S} 2$ and $\mathrm{S} 1$ and between the US and S1, thereby forming two independent temporal maps (with order and interval) between the paired events. Presumably, these temporal maps were integrated by superimposing the representations of the common element (i.e., S1) from the two phases of training. Combining the backward US-S1 temporal relationship and the S2-S1 relationship with a 5-s gap allowed S2 to predict an impending US, whereas having no gap between S2 and S1 did not. In the latter case, superimposing the maps would have caused the US to be expected simultaneously with the onset of S2, a relationship that is not conducive to appreciable behavioral control.

With the goal of refining the fourth tenet, regarding temporal integration, Molet et al. (2012) recently investigated exactly when temporal integration occurs by examining the effects of extinguishing S1 in Phase 3 prior to testing on S2. Figure 1 shows the design of the experiment, which was embedded within a sensory preconditioning (SPC) procedure. (In a separate experiment, in place of SPC, Molet et al. used of a secondorder conditioning [SOC] procedure in which Phases 1 and 2 were given in the reverse order, and they observed equivalent results.) They obtained evidence indicating that temporal integration occurs at the moment of testing rather than during Phase
2 training, at least with their procedures and parameters. They observed little or no conditioned suppression to S2 after extinction of S1, which suggested that the S1-alone presentations prior to testing on S2 disrupted the formation or expression of the integrated temporal relationship. On the basis of the view that temporal integration occurs at test, subjects presumably encoded the temporal relationships between stimuli in Phase 1 (i.e., S2-S1 in SPC, US-S1 in SOC) and the temporal relationship between stimuli in Phase 2 (i.e., US-S1 in SPC, S2-S1 in SOC), thereby forming two independent temporal relationships between the paired events. Finally, during an extinction treatment of S1, the subjects acquired a memory of S1 devoid of reinforcement (i.e., an extinction memory) that could interfere with expression of the associations to S1 that were acquired during Phases 1 and 2. The extinction memory of S1 presumably prevented $\mathrm{S} 2$ at test from reactivating the representation of the US mediated through the representation of S1. Consequently, S2 elicited little or no conditioned suppression. If the temporal memories of Phases 1 and 2 had been integrated during Phase 2, the resulting S2-US association with its own temporal attributes should not have been affected by the extinction memory of S1. This indicates that these temporal maps are stored as two independent associations (S2-S1 and US-S1) during the retention period between acquisition of the second association (i.e., Phase 2 ) and testing.

In a further effort to refine the fourth tenet of the TCH (i.e., temporal integration), our present goal was to assess likely associative structures following an initial test trial on which behavior indicative of integration was observed. More specifically, following an initial test trial, two possible associative 
structures could subsequently support behavioral control by S2: (1) Conditioned responding to S2 on subsequent tests could be the result of recurring integration of the two independently learned temporal maps, which remain independently stored in memory and are expressed as a chain of temporal maps at test (i.e., S2-S1 plus US-S1), just as seemingly occurred on the initial test trial; (2) temporal integration at the moment of initial testing could result in the formation of a direct S2-US (or S2-R, where R represents "response") association with its own temporal attributes.

These two different associative structures present very different theoretical interpretations concerning why the extinction of S1 prevented expression of temporal integration in the experiments by Molet et al. (2012). If temporal integration takes the form of a chain of associations carrying temporal information, then integration is not technically the formation of a new temporal map, but the use of multiple temporal maps joined by a common element that provides information about the occurrence of the biologically relevant event that drives the conditioned response. In the account based on a chain of associations, the extinction of S1 degrades or interferes with the critical link that predicts the US (S1-US), thereby undermining the certainty about whether or when the US will occur. Alternatively, if a new temporal map is formed at test, this new S2-US association should be resistant to any subsequent degradation of the S1-US association. Thus, extinction of S1 between a first and second test of S1 should have no effect on responding on the second test. If the latter result were to be observed, one might wonder why Molet et al. found evidence that an S2-US temporal map was not formed during Phase 2. There are several potential reasons why a new temporal map would not have formed during Phase 2 in the Molet et al. experiments, but we will defer discussion of these possibilities until the General Discussion. We simply note here that this question would not arise if the present experiments revealed that the associative structure responsible for responding to $\mathrm{S} 2$ did not change during an initial test of S2, because this account assumes that expression of integration is always dependent on the state of the two independent temporal maps (S2-S1 and US-S1) at the moment of testing. Although the TCH makes no distinction between the two structural forms of temporal integration described thus far, two experiments were conducted to identify the specific structure of temporal integration produced during an initial test on S2. With these considerations in mind, the following experiments were designed to elucidate the associative structure underlying temporal integration that would be observed when temporal integration occurred within SPC.

\section{Experiment 1}

Here we sought to determine the associative structure of integrated temporal relationships by using a modified version of
Molet et al.'s (2012) SPC design and a slightly different procedure (i.e., leverpress suppression and a brief test stimulus). Our goal was to determine whether responding to S2 following an initial test trial on S2 arises from an S2-S1-US chain of temporal maps (where the temporal relationship between S1 and the US is reversed-i.e., S2-S1 and US-S1), as Molet et al. found to be the case on an initial test of S2, or from an S2-US (or S2-R) temporal map. We addressed this question by extinguishing $\mathrm{S} 1$ after obtaining evidence that integration had occurred on an initial test of S2 (see Table 1). If integration during an initial test of S2 results in a novel direct S2-US (or S2-R) temporal map, then subsequent responding on a second test of $\mathrm{S} 2$ should be insensitive to extinction of $\mathrm{S} 1$ administered after integration has occurred. Presumably, S2 should be able to activate a temporally specified representation of the US (or R) without further mediation by S1. The extinction memory of $\mathrm{S} 1$ acquired during Phase 3 should result in subjects expecting neither US nor S2 to occur before S1. However, this new learning should not affect any previously acquired direct association between the representations of S2 and the US (or R) that was formed due to integration during Test 1. Accordingly, S2 should elicit strong conditioned suppression if tested again later (i.e., Test 2). In contrast, if extinction of $\mathrm{S} 1$ reduces subsequent responding to $\mathrm{S} 2$ after an initial test of S2, this would suggest that integration occurs over again on each test trial, with each integration being based on a chain of temporal maps, S2-S1-US (where the temporal relationship between $\mathrm{S} 1$ and the US is backward). In the latter case, extinction of S1 after the initial test of S2 should interfere with subsequent retrieval of the S2-S1 association (including its temporal component) and/or subsequent retrieval of the USS1 association (including its temporal component), which should result in a decrease in the effective integration between the S2-S1 association with its temporal relationship, and the US-S1 association with its respective temporal relationship. Note that this assumes that extinction is bidirectional, as was previously demonstrated with similar experimental designs by Arcediano, Escobar, and Miller (2005).

Experiment 1 included four groups (see Table 1). The central question was whether extinction of S1 after an initial test of S2

Table 1 Design of Experiment 1

\begin{tabular}{llllll}
\hline Groups & Phase 1 & Phase 2 & Test 1 & $\begin{array}{l}\text { Ph 3 } \\
\text { Ext }\end{array}$ & Test 2 \\
\hline Ext-Twice & S2_(5s)_S1 & US_(5s)_S1 & S2? & S1 & S2? \\
NoExt-Twice & S2_(5s)_S1 & US_(5s)_S1 & S2? & S3 & S2? \\
Ext-Once & S2_(5s)_S1 & US_(5s)_S1 & S3? & S1 & S2? \\
NoGap & S2(0s)S1 & US_(5s)_S1 & S2? & S3 & S2? \\
\hline
\end{tabular}

US = 1-s 1-mA footshock; S1, S2, and S3 are of 3-s duration; S2 = click; $\mathrm{S} 1$ and $\mathrm{S} 3=$ tone and white noise, counterbalanced; Ext = extinction; $\mathrm{Ph}=$ Phase 
in Group Ext-Twice ("Ext" refers to extinction of S1, and "Twice" refers to the fact that $\mathrm{S} 2$ is tested twice) would alter conditioned suppression to $\mathrm{S} 2$ on a second test relative to Group NoExt-Twice, in which extinction of S1 did not occur after the initial test of S2. Group NoExt-Twice was a control group included to demonstrate the occurrence of temporal integration at both tests with the present parameters. We also included two further control groups. Groups Ext-Once ("Once" meaning that $\mathrm{S} 2$ was tested once-i.e., only after extinction of S1) was designed to replicate Molet et al.'s (2012) findings that extinction of S1 prior to initial testing on S2 prevented integration from being observed. Finally, Group NoGap was intended to help demonstrate once again that temporal integration depends on its specific Phase 1 temporal relationship. Evidence of conditioned suppression in the NoGap condition would indicate that the temporal information was not relevant for any conditioned suppression to S2 observed in other groups. Given the similarity of the present parameters to those of Molet et al., this condition was expected to produce a low level of suppression appropriate for an innocuous stimulus. Specifically, this group was critical for demonstrating a replication of the finding that extinction of S1 prior to an initial test (i.e., in Group ExtOnce) prevents integration, or at least the expression of integration. During Test 1, Groups Ext-Twice and NoExt-Twice were used to demonstrate the integration of temporal maps relative to Group NoGap. The critical assessment occurred during Test 2, in which the effectiveness of the extinction treatment after integration that was expected to have occurred during Test 1 (Group Ext-Twice) was compared to extinction of S2 before integration occurred (Group Ext-Once).

\section{Method}

\section{Subjects}

The subjects were 24 male and 24 female, experimentally naive, Sprague-Dawley-descended rats purchased from Harlan (Indianapolis, IN). The mean body weights were $212 \mathrm{~g}$ for males (range $=198-221 \mathrm{~g}$ ) and $181 \mathrm{~g}$ for females (range $=170-203 \mathrm{~g}$ ). Subjects were randomly assigned to one of four groups: ExtTwice, NoExt-Twice, Ext-Once, and NoGap, all $n \mathrm{~s}=12$, counterbalanced for sex within groups. The animals were individually housed in a vivarium maintained on a 16:8-hr light:dark cycle. All experimental manipulations occurred near the middle portion of the light phase. The animals received free access to Purina Lab Chow, whereas water availability was limited to 30 min per day, following a progressive deprivation schedule initiated one week prior to the start of the study.

\section{Apparatus}

The apparatus consisted of 12 operant chambers, each measuring $30 \times 30 \times 27 \mathrm{~cm}(1 \times \mathrm{w} \times \mathrm{h})$. The side walls of the chamber were made of stainless steel sheet metal, and the front wall, back wall, and ceiling of the chamber were made of clear Plexiglas. The floor was constructed of 0.3-cm-diameter stainless steel rods, spaced $1.3 \mathrm{~cm}$ center to center. These rods permitted the delivery of a $1-\mathrm{s}, 1.0-\mathrm{mA}$ constant-current footshock that served as the US in the present experiment (parameters from Molet et al., 2012). Each chamber was housed in a separate environmental isolation chest and could be dimly illuminated by a house light (1.12-W, \#1820 incandescent bulb) mounted high on a wall of the experimental chamber. Each chamber was equipped with a Med Associates retractable lever $5 \mathrm{~cm}$ wide that could extend $2 \mathrm{~cm}$ into the chamber or could be retracted into a recess in a wall. Each chamber was equipped on the same wall with a solenoid liquid dispenser (Lafayette Instrument Co., Model No. 80201), which could deliver $0.04 \mathrm{ml}$ of water through the ceiling of a square recess $(3 \times 6 \times 6 \mathrm{~cm}, 1 \times \mathrm{w} \times \mathrm{h})$, which was located to the right of each lever. The house light was turned off for $0.5 \mathrm{~s}$ each time that water was delivered. The bottom of the recess, which contained a cup to catch the delivered water, was $3 \mathrm{~cm}$ above the floor of the chamber.

Ventilation fans in each enclosure provided a constant 76-dB (all auditory measures used the $\mathrm{C}$-scale) background noise. Three $45-\Omega$ speakers mounted on the interior right, left, and back sides of each environmental chest were used to deliver a click train $(6 \mathrm{~Hz})$, a complex tone (500 and $520 \mathrm{~Hz}$ presented simultaneously), and white noise. The click train served as S2, whereas the tone and white noise served as $\mathrm{S} 1$ and $\mathrm{S} 3$, counterbalanced. Each stimulus presentation had a duration of $3 \mathrm{~s}$ at $8 \mathrm{~dB}$ above the 76dB background (based on Molet et al., 2012).

\section{Procedure}

Shaping On Days 1-5, all subjects were shaped to leverpress on a variable-interval- (VI-)20 schedule for water reinforcement. Each shaping session was $60 \mathrm{~min}$ in duration. On Days 1 and 2, rats received continuous reinforcement as well as fixed-interval- (FI-)2-min delivery of water. Throughout the experiment, water delivery coincided with a 0.5 -s interruption of the house light to signal reinforcement. On Day 3, all animals were given only continuous reinforcement for each leverpress. Starting on Day 3, any animal that failed to make at least 100 leverpresses received additional hand-shaping. On Days 4 and 5, the VI-20 schedule was in force.

Phase 1 training On Days 6 and 7, all subjects were presented daily with six S2-S1 pairings during daily 60 -min sessions. For Groups Ext-Twice, NoExt-Twice, and Ext-Once, a 5-s gap separated the termination of S2 and the onset of S1. For Group NoGap, there was no gap; that is, S2 termination was followed immediately by S1 onset. The onsets of S2 occurred 6, 18, 28, 34,48 , and $56 \mathrm{~min}$ into the session. The lever was not available during Phase 1 training. 
Phase 2 training On Days 8 and 9, all subjects received four daily US-S1 pairings, with a 5-s gap between US onset and S1 onset. The US presentations occurred 9, 21, 36, and $51 \mathrm{~min}$ into the daily 60 -min sessions. The lever was not available during Phase 2 training.

Reshaping 1 On Days 10 and 11, all subjects were exposed once daily to the training context with the lever inserted for 60 min, to reestablish a steady rate of leverpressing before testing. The animals received water reinforcement based on a VI20 schedule.

Test 1 On Day 12, Groups Ext-Twice, NoExt-Twice, and NoGap were tested for conditioned leverpress suppression to S2, whereas Group Ext-Once was exposed to a neutral stimulus (S3). Stimulus presentation occurred $11 \mathrm{~min}$ into the 15 min test session. All stimuli were presented for $3 \mathrm{~s}$. A 3-s test stimulus is an unusually brief exposure for a test stimulus in a conditioned suppression study. This unusually brief test stimulus was necessary in order to accommodate Molet et al.'s (2012) training parameters that we used, as well as to avoid generalization decrement from the 3 -s CS of training to a longer test CS, which might have resulted in the animals treating the test stimulus as being different from the training CSs. Additionally, and most importantly, it also avoided behavioral extinction of S2 during the initial test, thereby allowing for a meaningful second test on S2 in the Twice conditions. Notably, we expected the short test CS, provided that it activated an expectation of an impending aversive US, to arouse fear that, once aroused, would continue to produce suppression for some time beyond the 3-s duration of the CS. This expectation was predicated on the sympathetic neurohormonal system being slow to return to its resting state after it has been activated. Test 2 could potentially have used a longer CS that would have been more typical for a leverpress suppression test; however, our doing so would have made direct comparisons across the two tests problematic. Therefore, we used identical test parameters (i.e., 3-s-duration CSs) in both Tests 1 and 2.

Suppression ratios were calculated using the rate of responding during the $120 \mathrm{~s}$ prior to $\mathrm{CS}$ presentation (the preCS score is denoted " $\mathrm{A}$ " here) and the $30 \mathrm{~s}$ following CS onset (the post-CS score is denoted "B") to produce Kamin suppression ratios of $4 \mathrm{~B} /(4 \mathrm{~B}+\mathrm{A})$. The longer pre-CS window was used to obtain a more accurate estimate of baseline leverpressing than would have been obtained by our using a 30-s window. No scores from Group Ext-Once during this test were included in any analysis of the Test 1 data, because S3 was both entirely novel and a physical stimulus (i.e., white noise or tone), as distinct from S1 (i.e., clicks). This group served as a control to assess whether the present preparation could conceptually replicate the behavior of Molet et al.'s (2012) original experimental group (discussed in the introduction) at Test 2.
Phase 3 training On Days 13 and 14, the subjects in Groups Ext-Twice and Ext-Once received 120 daily presentations of S1 alone during daily 60-min sessions, whereas the subjects in the remaining groups received 120 daily presentations of a novel stimulus (S3) alone during these sessions. The intertrial intervals between onsets of S1 or S3 were 10, 20, 30, 40, or $50 \mathrm{~s}$ (the mean intertrial interval was $30 \mathrm{~s}$ ). The lever was not available during Phase 3 training.

Reshaping 2 On Days 15 and 16, all subjects were exposed once daily to the training context with the lever inserted for 60 min, to reestablish a steady rate of leverpressing before testing. The animals received water reinforcement based on a VI20 schedule.

Test 2 On Day 17, all subjects were tested for leverpress suppression to S2. The testing was identical to that of Test 1 .

Results and discussion

Test 1

During testing, five animals were lost due to unusually low leverpress rates during the pre-CS period (two animals from Group Ext-Twice and three animals from NoExt-Twice). Groups Ext-Twice and NoExt-Twice were collapsed into a single "Integration" condition because they had received identical treatment up to this point in the experiment. A one-way analysis of variance (ANOVA) on leverpresses by Condition Integration and Group NoGap during the pre-CS period revealed no significant differences whether the five low responders were included, $F(1,34)<1$, or excluded, $F(1,29)=$ $1.71, p>.20$. The mean pre-CS scores were 17.26 for Condition Integration and 13.42 for Group NoGap.

A one-way ANOVA was conducted on the suppression ratio scores for Conditions Integration and NoGap (see Fig. 2). Animals in Condition Integration demonstrated more conditioned

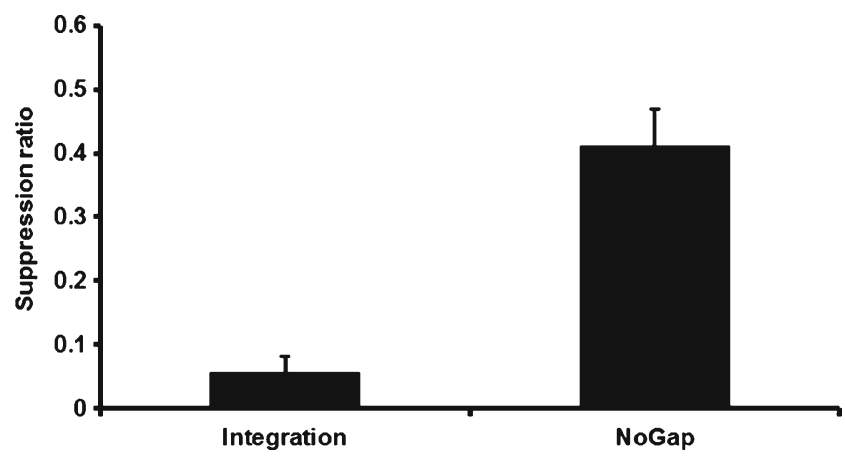

Fig. 2 Results of Experiment 1: Test 1. Mean suppression ratios to S2 for the pooled Integration condition and the NoGap condition. Error bars represent the standard errors of the means 
suppression than did those in Group NoGap, $F(1,29)=69.83$, $p<.05$, Cohen's $f=1.49$ (95\% confidence interval for $f[\mathrm{CI}]=$ $0.97,2.06)$. The results quite clearly replicated Groups Int and Control 1 from Molet et al.'s (2012, Exp. 1), but now using a leverpress suppression task, in contrast to Molet et al.'s lick suppression preparation. Thus, behavior indicative of the integration of associations acquired in Phases 1 and 2, including information about interstimulus intervals, was observed.

\section{Test 2}

A one-way ANOVA was conducted on the numbers of leverpresses emitted during the pre-CS period by the four different groups, and we found no significant differences in baseline leverpressing, $F<1$. The mean pre-CS scores were 28.92 for Group Ext-Twice, 28.42 for Group NoExt-Twice, 26.92 for Group Ext-Once, and 26.83 for Group NoGap. A similar ANOVA was conducted on the suppression ratios from all four groups (see Fig. 3). This analysis detected an effect of treatment, $F(3,44)=11.59, p<.05$, Cohen's $f=0.81(95 \% \mathrm{CI}=0.48$, 1.16). Planned comparisons using the error term from the ANOVA found that Group NoExt-Twice suppressed more than did Group NoGap, $F(1,44)=13.19, p<.05$, further supporting the interpretation that temporal integration of learning from Phases 1 and 2 had occurred. Additionally, Group Ext-Once exhibited less suppression than did Group NoExt-Twice, $F(1,44)=11.10, p<.05$, and did not differ from Group NoGap, $F<1$, which supports the conclusion that extinction of S1 prevented observable integration in Group Ext-Once at Test 2. Both Groups NoExt-Twice and NoGap received an additional exposure to S2 during Test 1, relative to Group Ext-Once. One might consider this a potential confound; however, the additional exposure to $\mathrm{S} 2$ would be expected to reduce behavioral control by $\mathrm{S} 2$ through extinction. Thus, if this potential confound had any effect, it would have yielded more behavioral control by S2 in Group Ext-Once, which is contrary to what was observed. Thus, the potential confound seems to have had little effect. These observations replicated the central findings of Molet et al. (2012). Most importantly, extinction of S1 was ineffective

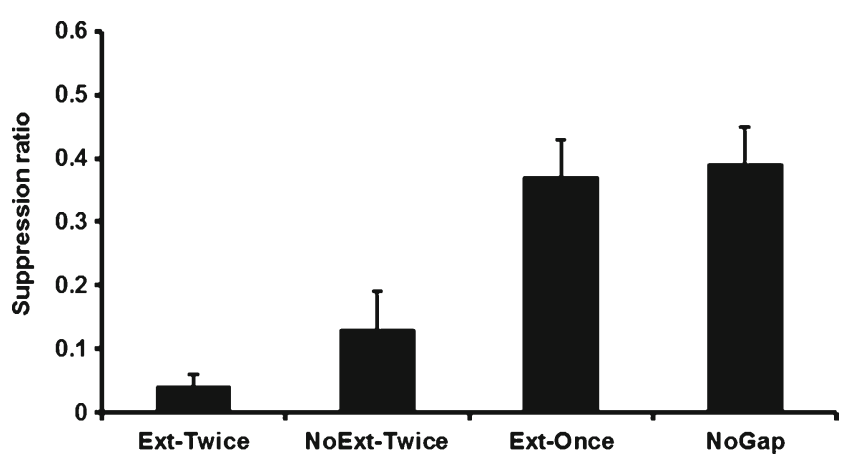

Fig. 3 Results of Experiment 1: Test 2. Mean suppression ratios to S2 for each group. Error bars represent the standard errors of the means in attenuating integration by Group Ext-Twice, which suppressed more than did Group Ext-Once, $F(1,44)=20.46$, $p<.05$, and did not differ from Group NoExt-Twice, $F(1,44)=$ $1.42, p>.23$.

Taken together, these results suggest that integration results in the formation of a direct association between $\mathrm{S} 2$ and the US (or the response). According to this interpretation, associations with $\mathrm{S} 1$ are necessary to mediate the initial integration of the maps at test, but they become unnecessary once integration has occurred. Essentially, S1 served to facilitate integration of the two temporal maps, S2-S1 and US-S1, into a new S2-US (or S2-R) map during an initial test trial. However, the question remained whether the learning that results from integration of memories at Test 1 is an $\mathrm{S}-\mathrm{S}$ or an S-R association. That is, temporal integration at Test 1 could have resulted in the formation of a direct S2-US association (i.e., an $S-S$ association), and/or $\mathrm{S} 2$ could have entered into a direct association with whatever output process governs the response elicited by $\mathrm{S} 2$ (i.e., an $S-R$ association).

\section{Experiment 2}

The goal of Experiment 2 was to determine whether responding at Test 2 in Experiment 1 was the result of an S2-US association or an S2-R association. Either association should remain able to produce a fear response after the extinction of S1, as each is independent of S1. Toward answering this question, we devalued the US between Tests 1 and 2, either with or without extinction of S1. US devaluation would reduce suppression if integration established an S2-US association, but not if integration established an S2-R association or both an S2-US association and an S2-R association. Thus, Experiment 2 was designed to test whether responding to S2 on Test 2 was dependent on the motivational value of the US at the time of testing. A number of parameters were changed in order to allow this manipulation, which also assessed the generality of our observations in Experiment 1. Specifically, in Experiment 2 we used a loud 3-s click train as the US instead of the 1.0-s footshock, in order to allow US devaluation through habituation, as prior research in our laboratory had found that rats exhibit considerable fear to initial presentations of the loud clicks, but exhibit habituation to them after a number of presentations (see, e.g., Laborda \& Miller, 2011). We also used slightly different stimuli, because using the click train as the US precluded our using it as a cue, and we used a longer trace between S2 and S1 because the loud clicks (i.e., the US) required a longer duration than did the footshock in order to induce appreciable fear.

Experiment 2 included six groups (see Table 2). The central question was whether devaluation of the US (through habituation) occurring after the initial test of S2 in Group ExtDev-Twice and Group NoExtDev-Twice ("Dev" refers to devaluation of the 
Table 2 Design of Experiment 2

\begin{tabular}{|c|c|c|c|c|c|c|c|}
\hline Groups & Phase 1 & Phase 2 & Test 1 & $\begin{array}{l}\mathrm{Ph} 3 \\
\text { Ext }\end{array}$ & $\begin{array}{l}\mathrm{Ph} 4 \\
\text { Dev }\end{array}$ & Test 2 & Test US \\
\hline Ext-Twice & $\mathrm{S} 2 \_(6 \mathrm{~s}) \_\mathrm{S} 1$ & US__(6s)_S 1 & $\mathrm{~S} 2 ?$ & S1 & Ctx & $\mathrm{S} 2 ?$ & US? \\
\hline NoExt-Twice & $\mathrm{S} 2$ __(6s)_S 1 & US_ $(6 \mathrm{~s}) \_\mathrm{S} 1$ & S2? & S3 & Ctx & S2? & US? \\
\hline ExtDev-Twice & $\mathrm{S} 2 \_(6 \mathrm{~s}) \_\mathrm{S} 1$ & US_ $(6 s) \_S 1$ & S2? & S1 & $60 \mathrm{US}$ & S2? & US? \\
\hline NoExtDev-Twice & $\mathrm{S} 2$ __(6s)_S 1 & US_ $(6 s) \_S 1$ & S2? & S3 & $60 \mathrm{US}$ & $\mathrm{S} 2 ?$ & US? \\
\hline Ext-Once & $\mathrm{S} 2$ __(6s)_S 1 & US_ $(6 \mathrm{~s}) \_\mathrm{S} 1$ & S3? & S1 & Ctx & S2? & US? \\
\hline NoGap & $\mathrm{S} 2(0 \mathrm{~s}) \mathrm{S} 1$ & US_ $(6 s) \_S 1$ & S2? & S3 & Ctx & S2? & US? \\
\hline
\end{tabular}

All stimuli (S1, S2, S3, and US) were presented for $3 \mathrm{~s}$. US = loud clicks; S2 = tone; S1 and S3 = white noise and SonAlert, counterbalanced; Ext = extinction; $\mathrm{Dev}=$ devaluation of unconditioned stimulus; $\mathrm{Ph}=$ phase

US) would alter conditioned suppression to S2 on a second test, relative to Groups Ext-Twice and NoExt-Twice, in which the US was not devalued. The latter two groups were exposed to the context alone during the devaluation phase, which should have had no particular effect on responding to S2. Group NoExtTwice was a control group to demonstrate the occurrence of temporal integration with the new parameters. As in Experiment 1, we included two further control groups, Group Ext-Once and Group NoGap, for the same reasons discussed in Experiment 1.

During Phase 1, all groups except Group NoGap received S2-S1 pairings with a 6-s gap between the termination of S2 and the onset of S1, whereas Group NoGap received S2-S1 pairings with no gap. During Phase 2, S1 was backwardpaired with the click-train US (i.e., US-S2) for all six groups, with a 6-s gap between the onsets of the US and of S2. At Test 1, all groups except Group Ext-Once were tested for conditioned suppression to S2, whereas Group Ext-Once was presented with an irrelevant stimulus, S3. During Phase 3, rats in Groups Ext-Twice, ExtDev-Twice, and Ext-Once received presentations of $\mathrm{S} 1$ alone. Rats in the remaining groups received presentations of S3 (the irrelevant stimulus) alone. In Phase 4, rats in Groups ExtDev-Twice and NoExtDev-Twice received presentations of the US alone (i.e., operational devaluation of the US), whereas the rats in the remaining groups received equivalent context exposure. At Test 2, all six groups were tested for conditioned suppression to S2. Finally, to assess the efficacy of the US devaluation treatment, all rats were tested for unconditioned suppression to the US.

Of central interest was the suppression to $\mathrm{S} 2$ at Test 2 by Groups ExtDev-Twice and NoExtDev-Twice, both of which received devaluation of the US in Phase 4 after the initial test of S2. If the temporal integration at Test 1 resulted in the creation of a direct S2-US association, the devaluation of the US after the initial test of S2 should attenuate conditioned suppression on Test 2 . However, such an effect should not be observed if temporal integration at Test 1 resulted in the formation of a direct $\mathrm{S} 2-\mathrm{R}$ association. The predictions regarding the remaining groups were the same as those we described for the analogous groups in Experiment 1. In fact, the remaining groups closely corresponded to the ones in
Experiment 1, the only differences being that (a) a fourth phase was added, which was used to equate context exposure across groups and presumably had no effect on responding to S2; (b) the 3-s loud click replaced the 1.0-s footshock; (c) the physical stimuli were changed; and (d) the gaps between stimuli in Phases 1 and 2 were increased to $6 \mathrm{~s}$ from $5 \mathrm{~s}$.

Method

\section{Subjects}

The subjects were 36 male (190-223 g) and 36 female (174$203 \mathrm{~g}$ ) experimentally naive Sprague--Dawley-descended rats purchased from Harlan (Indianapolis, IN). The rats were randomly assigned to one of six groups: Ext-Twice, NoExtTwice, ExtDev-Twice, NoExtDev-Twice, Ext-Once, and NoGap, all $n \mathrm{~s}=12$, counterbalanced for sex within groups. One subject in Group NoExt-Twice was eliminated prior to Test 1 due to illness. The rats were housed and maintained as in Experiment 1.

\section{Apparatus}

The apparatus and the stimuli were identical to those described in Experiment 1, except for the following changes. A SonAlert was mounted inside each chamber. The SonAlert could produce a $1900-\mathrm{Hz}$ tone $8 \mathrm{~dB}$ above background. The loud click train, presented for $3 \mathrm{~s}$ at $30 \mathrm{~dB}$ above background, served as the US. The tone served as S2, and the white noise and SonAlert served as S1 and S3, counterbalanced within groups.

\section{Procedure}

Shaping The leverpress shaping was identical to that of Experiment 1 .

Phase 1 training This phase was similar to the Phase 1 training in Experiment 1, except for the following changes. For Groups Ext-Twice, NoExt-Twice, ExtDev-Twice, NoExtDev-Twice, and Ext-Once, a 6-s gap was inserted between the termination 
of S2 and the onset of S1. For Group NoGap, there was no gap; that is, $\mathrm{S} 2$ termination was followed immediately by $\mathrm{S} 1$ onset.

Phase 2 training On Days 8 and 9, all subjects received two daily US-S1 pairings, with a 6-s gap between US onset and S1 onset. US presentations occurred 21 and $45 \mathrm{~min}$ into a daily 60-min session. The lever was not available during Phase 2 training.

Reshaping 1 On Days 10 and 13, all subjects were exposed once daily to the training context with the lever inserted for 60 $\mathrm{min}$, to reestablish a steady rate of leverpressing before testing. The animals received water reinforcement based on a VI20 schedule. The experiment was placed on a two-day hiatus on Days 11 and 12. Animals continued to be water deprived, but remained in their home cages on these days.

Test 1 On Day 14, Groups Ext-Twice, NoExt-Twice, ExtDevTwice, NoExtDev-Twice, and NoGap were tested for conditioned leverpress suppression to S2, whereas Group Ext-Once was tested for leverpress suppression to a neutral stimulus, S3. Stimulus presentation was initiated $11 \mathrm{~min}$ into the 15 -min test session. As during training, all stimuli were presented for $3 \mathrm{~s}$. Kamin suppression ratios were calculated as in Experiment 1.

Phase 3 treatment On Days 15 and 16, subjects in Groups Ext-Twice, ExtDev-Twice, and Ext-Once received 120 daily presentations of $\mathrm{S} 1$ alone during daily 60 -min sessions, whereas subjects in the remaining groups received 120 daily presentations of a novel neutral stimulus (S3) alone. The intertrial intervals between the onsets of $\mathrm{S} 1$ and $\mathrm{S} 3$ were 10 , $20,30,40$, or $50 \mathrm{~s}$ (mean intertrial interval $=30 \mathrm{~s}$ ). The lever was not available during Phase 3 training.

Phase 4 treatment On Days 17 and 18, the subjects in Groups ExtDev-Twice and NoExtDev-Twice received 30 daily 3-s presentations of the US alone during daily 60 -min sessions, whereas the subjects in the remaining groups received equivalent context exposure. The lever was not available during Phase 4 training.

Reshaping 2 On Days 19 and 20, all subjects were exposed once daily to the training context with the lever inserted for 60 $\mathrm{min}$, to reestablish a steady rate of leverpressing before testing. The animals received water reinforcement based on a VI20 schedule.

Test 2 On Days 21 and 22, all subjects were tested for leverpress suppression to S2. Testing was identical to that during Test 1 , with the exception that $\mathrm{S} 2$ was presented to the subjects in all six groups. A second test day with S2 (Day 22) was added to verify the results of Day 21 , considering the somewhat surprising observations on Day 21 (see below). The behavior on Day 22 closely replicated that on Day 21; hence, the data from Days 21 and 22 were pooled. The combined statistics (suppression ratios calculated for each subject using the summed pre-CS scores and summed CS scores from Days 21 and 22) closely paralleled the observations of Test Day 21 alone; therefore, only the combined statistics are described in the following Results section.

Reshaping 3 On Day 23, all subjects were exposed once to the training context with the lever inserted for $60 \mathrm{~min}$, to reestablish a steady rate of leverpressing before further testing. The animals received water reinforcement based on a VI-20 schedule.

Test US On Day 24, all subjects were tested for leverpress suppression to the US. Testing was identical to that during Test 1, with the exception that the US was presented to each subject. One rat from Group ExtDev-Twice failed to respond during the 120-s pre-CS period and was removed from the subsequent analysis, because a suppression ratio could not be calculated.

Results and discussion

Examining analogous groups, less suppression was consistently observed in Experiment 2 than in Experiment 1. This presumably was due to the change in stimuli and parameters, particularly the substitution of a loud click train in Experiment 2 in place of the footshock that had been used in Experiment 1.

\section{Test 1}

Given their identical treatment up to this point, Groups ExtTwice, NoExt-Twice, ExtDev-Twice, and NoExtDev-Twice were initially pooled to form a single Integration condition to be compared with Group NoGap (see Fig. 4). A one-way ANOVA was conducted on the numbers of leverpresses emitted

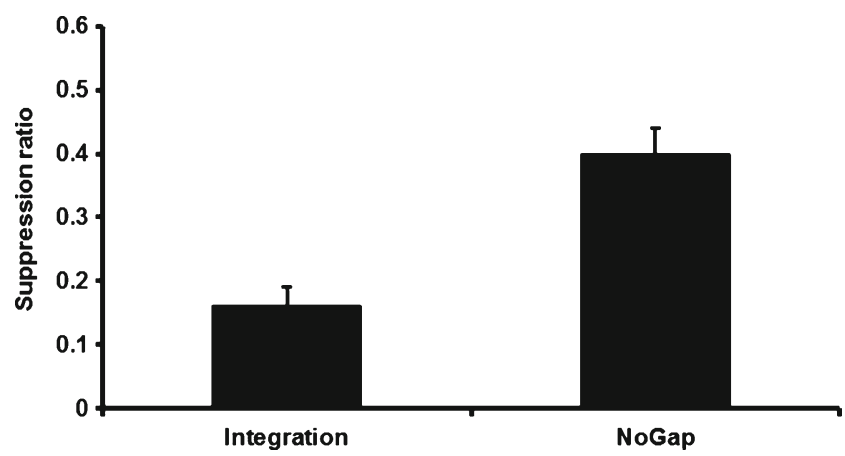

Fig. 4 Results of Experiment 2: Test 1. Mean suppression ratios to S2 for the collapsed "Integration" condition and the NoGap condition. Error bars represent the standard errors of the means 
during the pre-CS period, and it did not reveal a reliable difference between Condition Integration and Group NoGap, $F(1,57)=3.80, p>.05$. The mean pre-CS scores were 23.02 for Condition Integration and 29.50 for Group NoGap. A similar ANOVA was conducted on the suppression ratios for Condition Integration and Group NoGap. Condition Integration exhibited more suppression than did Group NoGap, $F(1,57)=$ $16.23, p<.05$, Cohen's $f=0.52(95 \% \mathrm{CI}=0.25,0.80)$. This replicates prior demonstrations of differences in conditioned behavior as a result of temporal integration (e.g., Exp. 1). A subsequent one-way ANOVA conducted on the four test-twice groups revealed no significant difference among the groups, $F<1$ (see Fig. 5). The mean PreCS scores were 21.25 for Group Ext-Twice, 20.92 for Group NoExt-Twice, 24.92 for Group ExtDev-Twice, and 25.83 for Group NoExtDev-Twice.

\section{Test 2}

One-way ANOVAs were conducted on the pre-CS scores and on the combined suppression ratios across Days 21 and 22 (see Fig. 6). We found no significant effect of group on the numbers of leverpresses emitted during the pre-CS period, $F<1$. The mean pre-CS scores were 29.33 for Group Ext-Twice, 27.55 for Group NoExt-Twice, 30.88 for Group ExtDev-Twice, 30.17 for Group NoExtDev-Twice, 27.17 for Group Ext-Once, and 29.71 for Group NoGap. An effect of group was detected on the combined suppression ratios, $F(5,65)=6.29, p<.05$, Cohen's $f=0.61(95 \% \mathrm{CI}=0.34,0.89)$. Planned contrasts were conducted to replicate the analysis of Experiment 1 among Groups NoGap, Ext-Once, Ext-Twice, and NoExt-Twice. These planned contrasts showed that Group NoExt-Twice suppressed more than did Group NoGap, $F(1,65)=19.00, p<.05$, indicating that integration had occurred and was observable during Test 2. Group Ext-Once showed less suppression than did Group NoExt-Twice, $F(1,65)=19.14, p<.05$, demonstrating that extinction of $\mathrm{S} 1$ disrupted temporal integration, or at least its expression. Group Ext-Once was not reliably different from Group NoGap, $F<1$, supporting the conclusion that integration was observably absent when S2 was tested only after

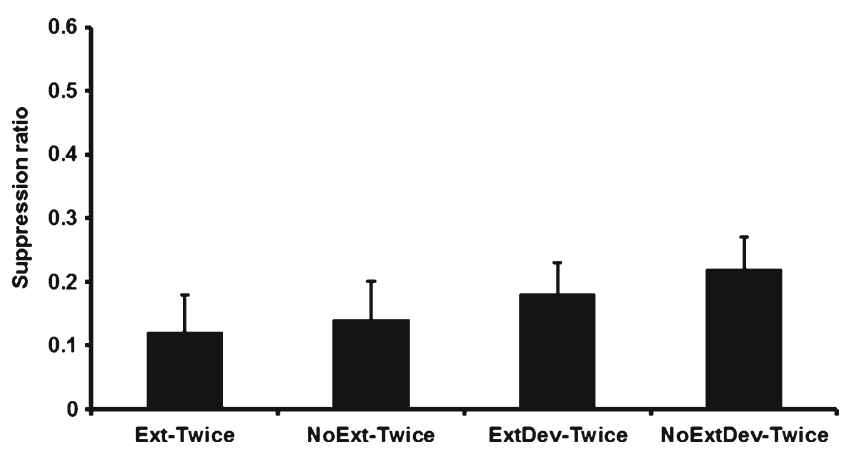

Fig. 5 Results of Experiment 2: Test 1. Mean suppression ratios to S2 for each group forming the collapsed "Integration" condition. Error bars represent the standard errors of the means

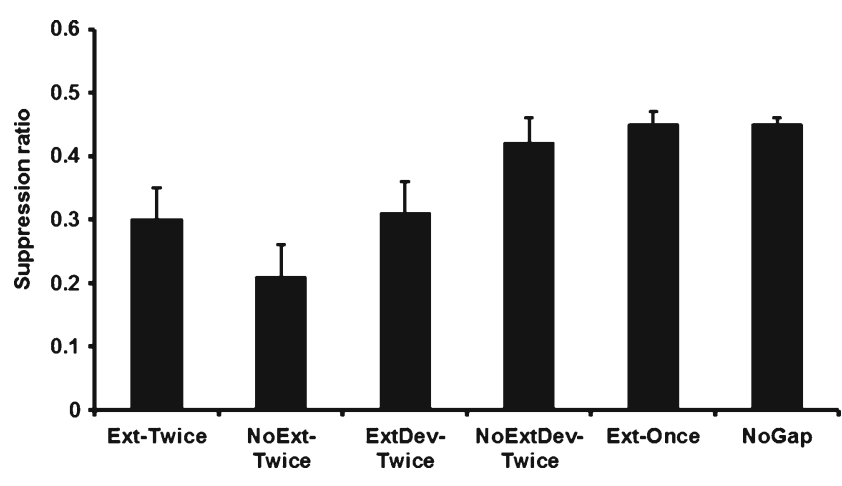

Fig. 6 Results of Experiment 2: Test 2. Mean suppression ratios to S2 for each group. Error bars represent the standard errors of the means

extinction of S1. As in Experiment 1, Groups NoExt-Twice and NoGap had been previously tested on S2, whereas Group ExtOnce had not, which introduced a potential confound due to the 3 -s presentation of $\mathrm{S} 2$ on the first test. But the likely effect of this potential confound would be relatively more suppression to S2 in Group Ext-Once, which was not observed. Moreover, Group Ext-Twice showed more suppression than did Group Ext-Once, $F(1,65)=7.60, p<.05$, which indicates that integration was not disrupted by extinction of S1 once integration of the temporal maps had taken place during Test 1. Finally, Group ExtTwice did not differ from Group NoExt-Twice, $F(1,65)=$ $2.62, p>.05$, which further supports the conclusion that once integration had taken place, subsequent responding to S2 was no longer dependent on associations with S1. As in Experiment 1, integration during Test 1 appeared to have resulted in the formation of a novel association with S2. All this replicated Experiment 1, despite the procedural differences between the two experiments, which speaks to the reliability and robustness of the present effects.

To address Experiment 2's central question of whether temporal integration resulted in the formation of an S2-US association (an S-S account) or an association in which S2 was able to elicit a fear response without activating a representation of the US (i.e., an S-R account), a 2 (Dev vs. NoDev) $\times 2$ (Ext vs. NoExt) ANOVA on the four groups in the Twice condition was conducted. A significant interaction was detected, $F(1,65)=$ $6.04, p<.05$. Planned contrasts were conducted to identify the source of this interaction. Group Ext-Twice did not significantly differ from Group ExtDev-Twice, $F<1$, which suggests that conditioned responding to S2 was driven by an S2-R association rather than an S2-US association. If responding following extinction of S1 was the product of an S2-US association, then Group ExtDev-Twice should have shown attenuated suppression relative to Group Ext-Twice once the US was devalued, which was not the case. Additionally, no significant difference was observed between Groups Ext-Twice and NoExt-Twice, $F(1,65)=2.62, p>.11$, which is consistent with our observations in Experiment 1 and suggests that extinction of S1 after an initial test of S2 has little effect upon subsequent suppression to 
S2. A peculiar aspect of this interaction was that if S1 was not extinguished (Group NoExt-Twice), conditioned suppression to S2 was attenuated by the devaluation treatment (Group NoExtDev-Twice), $F(1,65)=13.49, p<.05$. Thus, without extinction of S1, conditioned suppression to S2 seems to have been driven by an S2-US association.

Collectively, these findings suggest that the extinction treatment following Test 1 changed the basis of responding on Test 2 from S2-US to S2-R. This is surprising, given that integration seemingly occurred during Test 1 . We speculate that in the Twice condition, both S2-US and S2-R associations were formed during Test 1, with the S2-US association inhibiting expression of the S2-R association on Test 2 regardless of whether the US was devalued (Group NoExtDevTwice) or not (Group NoExt-Twice). However, the S2-US association did not support suppression if the US had been devalued (Group NoExtDev-Twice). In contrast, extinction of $\mathrm{S} 1$ seems to have released the S2-R association from inhibition, thereby allowing $\mathrm{S} 2$ to elicit suppression, regardless of the motivational status of the US. Admittedly, this speculative account is at best incomplete, as it fails to explain why extinction of $\mathrm{S} 1$ has this effect, and at worst is simply wrong. We offer it as no more than food for thought. But, despite our lack of a complete and compelling account, the data are clear in demonstrating that responding to $\mathrm{S} 2$ following extinction of S1 (after integration has occurred on Test 1) is based on an $\mathrm{S} 2-\mathrm{R}$ association, whereas without extinction of $\mathrm{S} 1$, it is based on an S2-US association.

\section{Test US}

A one-way ANOVA was conducted on the pre-CS scores and on the suppression ratios from Day 24. No significant effect of group was observed on the numbers of response emitted during the pre-CS period, $F<1$. The mean pre-CS scores were 27.25 for Group Ext-Twice, 29.75 for Group NoExtTwice, 31.36 for Group ExtDev-Twice, 25.45 for Group NoExtDev-Twice, 27.00 for Group Ext-Once, and 31.25 for Group NoGap. An effect of group was observed when the suppression ratios (see Fig. 7) were analyzed, $F(5,64)=$ 18.55, Cohen's $f=1.12(95 \% \mathrm{CI}=0.81,1.44)$ : The US devaluation treatment was shown to reduce suppression to the US. The two US devaluation groups (Groups ExtDevTwice and NoExtDev-Twice) showed less suppression than did each of the other groups, which did not receive devaluation treatment, lowest $F(1,64)=23.77, p<.05$. Additionally, Groups ExtDev-Twice and NoExtDev-Twice did not differ from each other, $F<1$, and differences among the groups that did not receive devaluation treatment were not significant, highest $F(1,64)=1.08, p>.25$. These results validate the devaluation treatment, showing that it was effective and did not differentially affect responding to the US between Groups ExtDev-Twice and NoExtDev-Twice.

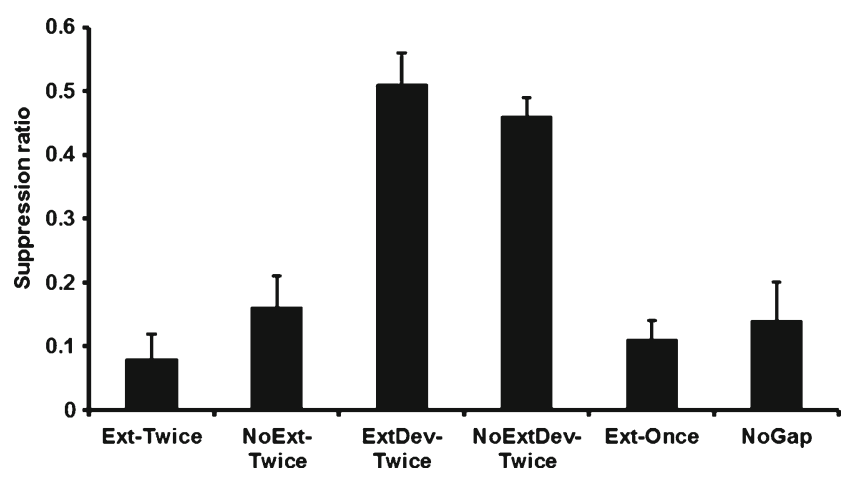

Fig. 7 Results of Experiment 2: Test US. Mean suppression ratios to US for each group. Error bars represent the standard errors of the means

In sum, we replicated the finding of Molet et al. (2012) that integration occurs at the time of testing using a somewhat different preparation (i.e., a different US and a longer trace between $\mathrm{S} 2$ and S1). Experiment 2 also replicated the finding of Experiment 1, which ruled out a chaining account of the integrated representation following Test 1 by showing that responding on Test 2 was unaffected by extinction of S1 if integration had been demonstrated on Test 1. Finally, Experiment 2 suggests that the new association(s) resulting from integration is(are) some sort of hybrid of S2-US and S2-R. Without extinction of S1, S2 appears to be aversive, independent of any association with $\mathrm{S} 1$ or the US. This was illustrated by responding in the Ext condition having been unaffected by the US devaluation treatment. Surprisingly, responding to S2 appears to be driven by an S-R association only if extinction of S1 has occurred; otherwise, responding was dependent on the motivational status of the US. In the NoExt condition, US devaluation did attenuate suppression to S2. Although the empirical results are quite clear, the specific mechanism by which exposure to $\mathrm{S} 1$ shifts responding to $\mathrm{S} 2$ from $\mathrm{S}-\mathrm{S}$ to $\mathrm{S}-\mathrm{R}$ is beyond the scope of the present research, and alternative accounts are certainly possible. Regarding the question of whether integration took the form of S-S or S-R learning, it tentatively appears that both types of learning took place, with specific conditions determining which would be expressed (e.g., Holland, 2008).

\section{General discussion}

Experiment 1 was designed to replicate the findings of Molet et al. (2012) using a slightly different procedure (i.e., leverpress suppression and brief test stimulus) and, critically, to determine whether in SPC an initial test of S2 was sufficient to establish a new S2-US association that was no longer dependent on S1 (i.e., a direct S2-US association). The results confirmed previous findings that extinction of S1 prior to initial testing on S2 prevented integration from being observed, a finding consistent with that of Rizley and Rescorla 
(1972), who used an SPC design that precluded sensitive assessment of temporal integration. However, if extinction of $\mathrm{S} 1$ was delayed until after an initial test of $\mathrm{S} 2$, this same extinction treatment was ineffective in attenuating behavior indicative of integration. Conditioned suppression was just as robust as if no extinction of S1 had occurred. These findings suggest that temporal integration at the moment of testing takes the form of establishing a new direct S2-US association. After integration has occurred, conditioned suppression to S2 appeared to be entirely independent of the associative status of $\mathrm{S} 1$. However, one additional possibility was that integration might take the form of a direct S2-R association rather than a direct S2-US association. This was addressed in the second experiment.

Experiment 2 again replicated the finding of Molet et al. (2012) that integration occurs at the time of testing, using slightly different parameters than were used in Experiment 1. Experiment 2 also replicated the finding of Experiment 1, which ruled out a chaining account of integration by showing that responding on Test 2 was unaffected by extinction of S1 after integration had been demonstrated during an initial test. Unexpectedly, responding to S2 appeared to be driven by an S-R association only if extinction of S1 had occurred; otherwise, responding was dependent on the motivational status of the US. US devaluation did attenuate conditioned suppression to S2 if extinction of S1 had not occurred. These results suggest that extinction of $\mathrm{S} 1$ shifts responding to $\mathrm{S} 2$ from $\mathrm{S}-$ $\mathrm{S}$ to $\mathrm{S}-\mathrm{R}$, although the specific mechanism for this shift in associative structure remains unclear. We can safely conclude that temporal integration does result in the formation of a new direct S2-US or direct S2-R association, even though this new association may not be the only determinant of responding. Further investigations should examine the conditions that favor the formation and/or expression of S-S or S-R learning, rather than simply asking whether learning is $\mathrm{S}-\mathrm{S}$ or $\mathrm{S}-\mathrm{R}$ learning. The procedure used in the present Experiment 2 might be useful in illuminating these conditions.

One might well ask why integration seemingly occurred during the initial test trial with S2 but not during Phase 2. After all, during Phase 2 the US was presented, and presentation of $\mathrm{S} 1$ could have activated the representation of S2. Thus, representations of S2 and the US, and their individual temporal maps relative to $\mathrm{S} 1$, were likely active at the same time, a situation that might have resulted in the establishment of an S2-US (and/or S2-R) association (Hall, 1996; Holland, 1990). However, the present data clearly demonstrate that the establishment of a direct S2-US association did not occur during Phase 2, but did occur during the first test with $\mathrm{S} 2$. The question is what makes the first test different from Phase 2. At least four possibilities can be considered. First, S1 was presented during Phase 2, whereas S2 was presented during the first test. Thus, activation of the representation of S2 was retrieval-generated during Phase 2 but was self-generated during the first test of S2. Learning is likely quicker for selfgenerated than for retrieval-generated representations (e.g., Wagner, 1981). Second, the process of forming an S1-US association during Phase 2 may have interfered with either activation of the S2 representation or establishment of an S2 US association. Third, the US was presented during Phase 2, whereas it was not presented on the first test. Thus, activation of the US representation was self-generated during Phase 2 but retrieval-generated during the first test with S2. Consequently, the US presentation during Phase 2 may have interfered with $\mathrm{S} 1$ activating the representation of S2 during Phase 2. Fourth, some information processing that occurred during Phase 2 may have set the necessary conditions (e.g., formation of the US-S1 association) for subsequent establishment of a direct S2-US (and/or S2-R) association during the first test. For example, the second test occurred some days after Phase 2, giving what was learned in both Phases 1 and 2 more time to consolidate, which might be a necessary condition for memory integration. If we knew which of these options was the critical factor, we could then speculate why. Neither Molet et al. (2012) nor the present research was designed to determine which of these factors was critical. However, the second-order conditioning experiments conducted by Molet et al., with Phase 2 consisting of S2-S1 pairings, challenge the first and third accounts, because S1 was physically present during Phase 2 and the biologically relevant US was not. Account 4 might be salvaged by stating that the mere presence of two stimuli was sufficient to disrupt integration during Phase 2. At this point, we may conclude that, for whatever reason, the data are unambiguous in showing that enduring integration occurred during the first test of S2, but not during Phase 2.

To date, experiments designed in the framework of the TCH have clearly shown that when two independent temporal relationships (e.g., S2-S1 and S1-US) are learned and have a common element (here, S1), they are integrated through superimposing the two representations of the common element, thereby creating a temporal relationship between the two unique elements (S2 and the US, in the present case) that had never actually been paired. Putting our present observations into a larger perspective, these results allowed us to elaborate on recent refinements of the TCH concerning (a) the moment of temporal integration, which presumably occurs during testing (Molet et al., 2012); (b) the associative structure of temporal integration, which takes the form of a new S2-US (and/or S2-R) association (our present data); (c) the directional nature of the temporal coding, which has been found to take the form of a single, bidirectional link (Arcediano et al., 2005); and (d) the modulation by contextual cues of ambiguous temporal relationships (Molet, Urcelay, Miguez, \& Miller, 2010). The TCH makes clear, testable, a priori predictions, many of which have been confirmed. Although it makes only qualitative predictions (i.e., the rank ordering of responding among conditions), we believe that at this point in time, the 
fourth tenet of the $\mathrm{TCH}$ (i.e., integration) now provides a clearer description of how subjects encode and integrate interval relationships.

Author note The research was supported by NIMH Grant No. 33881. G.M. was supported by the Comision Nacional de Ciencia y Tecnologia (CONICYT-Chile) and the Fulbright Program. The authors are grateful to Cara Burney, Lisa Mash, and Julia Soares for comments on an earlier version of the manuscript.

\section{References}

Arcediano, F., Escobar, M., \& Miller, R. R. (2003). Temporal integration and temporal backward associations in humans and nonhuman subjects. Learning \& Behavior, 31, 242-256.

Arcediano, F., Escobar, M., \& Miller, R. R. (2005). Bidirectional associations in humans and rats. Journal of Experimental Psychology: Animal Behavior Processes, 31, 301-318. doi:10.1037/0097-7403. 31.3.301

Arcediano, F., \& Miller, R. R. (2002). Some constraints for models of timing: A temporal coding hypothesis perspective. Learning and Motivation, 33, 105-123.

Hall, G. (1996). Learning about associatively activated stimulus representations: Implications for acquired equivalence in perceptual learning. Animal Learning \& Behavior, 24, 233-255. doi:10.3758/ BF03198973
Holland, P. C. (1990). Event representation in Pavlovian conditioning: Image and action. Cognition, 37, 105-131.

Holland, P. C. (2008). Cognitive versus stimulus-response theories of learning. Learning \& Behavior, 36, 227-241. doi:10.3758/LB.36.2.227

Honig, W. K. (1981). Working memory and the temporal map. In N. E. Spear \& R. R. Miller (Eds.), Information processing in animals: Memory mechanisms (pp. 167-197). Hillsdale, NJ: Erlbaum.

Laborda, M. A., \& Miller, R. R. (2011). S-R associations, their extinction, and recovery in an animal model of anxiety: A new associative account of phobias without recall of original trauma: S-R associations, their extinction, and recovery. Behavior Therapy, 42, 153-169.

Matzel, L. D., Held, F. P., \& Miller, R. R. (1988). Information and expression of simultaneous and backward associations: Implications for contiguity theory. Learning and Motivation, 19, 317-344.

Molet, M., Miguez, G., Cham, H. X., \& Miller, R. R. (2012). When does integration of independently acquired temporal relationships take place? Journal of Experimental Psychology: Animal Behavior Processes, 38, 369-380.

Molet, M., Urcelay, G. P., Miguez, G., \& Miller, R. R. (2010). Using context to resolve temporal ambiguity. Journal of Experimental Psychology: Animal Behavior Processes, 36, 126-136.

Rizley, R. C., \& Rescorla, R. A. (1972). Associations in second-order conditioning and sensory preconditioning. Journal of Comparative and Physiological Psychology, 81, 1-11.

Savastano, H. I., \& Miller, R. R. (1998). Time as content in Pavlovian conditioning. Behavioural Processes, 44, 147-162.

Wagner, A. R. (1981). SOP: A model of automatic memory processing in animal behavior. In N. E. Spear \& R. R. Miller (Eds.), Information processing in animals: Memory mechanisms (pp. 5-47). Hillsdale, NJ: Erlbaum. 